\title{
Fabrication and Characterization of Woodpile Structures for Direct Laser Acceleration
}

\author{
C. McGuinness*, , E. Colby*, R.J. England*, J. Ng*, R.J. Noble*, E. Peralta*, ${ }^{*}$, K. \\ Soong*, ${ }^{*, \dagger}$ J. Spencer*, D. Walz ${ }^{*}$ and R.L. Byer ${ }^{\dagger}$ \\ *SLAC National Accelerator Laboratory, 2575 Sand Hill Rd. Melo Park, CA 94025 \\ ${ }^{\dagger}$ E.L. Ginzton Laboratory, Nano Center Building, 348 Via Pueblo Mall, Stanford, CA 94305-4088
}

\begin{abstract}
An eight and nine layer three dimensional photonic crystal with a defect designed specifically for accelerator applications has been fabricated. The structures were fabricated using a combination of nanofabrication techniques, including low pressure chemical vapor deposition, optical lithography, and chemical mechanical polishing. Limits imposed by the optical lithography set the minimum feature size to $400 \mathrm{~nm}$, corresponding to a structure with a bandgap centered at $4.26 \mu \mathrm{m}$. Reflection spectroscopy reveal a peak in reflectivity about the predicted region, and good agreement with simulation is shown. The eight and nine layer structures will be aligned and bonded together to form the complete seventeen layer woodpile accelerator structure.
\end{abstract}

Keywords: Direct Laser Acceleration, Three-Dimensional Photonic Crystal, Woodpile Structure, dielectric, lithography, nanofabrication, fourier transform infrared spectroscopy, FTIR

PACS: $41.75 . J v$

\section{INTRODUCTION}

Laser driven dielectric accelerator structures have recently become of interest due to the potential GeV/m accelerating gradients [1,2], widespread availability of high power lasers, low costs associated with large scale fabrication of lithographic structures, and attosecond bunch lengths in such accelerating schemes $[3,4,5]$. High loss in metals at optical frequencies prompts exploration of alternate means for confinement and manipulation of the electromagnetic (EM) fields used for laser acceleration. Photonic crystals offer an attractive way to confine laser radiation, allowing for the manipulation of the EM fields without the use of metallic boundaries. One structure that has been explored in some detail, but only theoretically for particle accelerator applications, is the woodpile structure, shown in Figure 1 (a). This structure is attractive because it has a fully three-dimensional bandgap, is amenable to common lithographic procedures, and allows for much flexibility in structure refinement and modification of particular features, such as couplers or focusing elements.

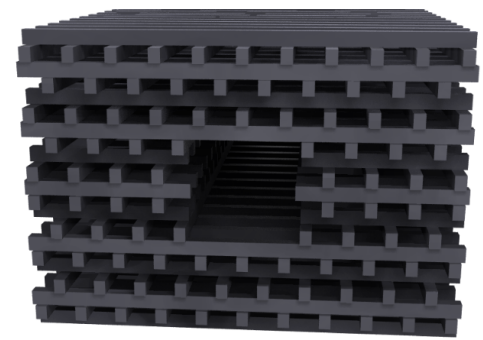

(a)

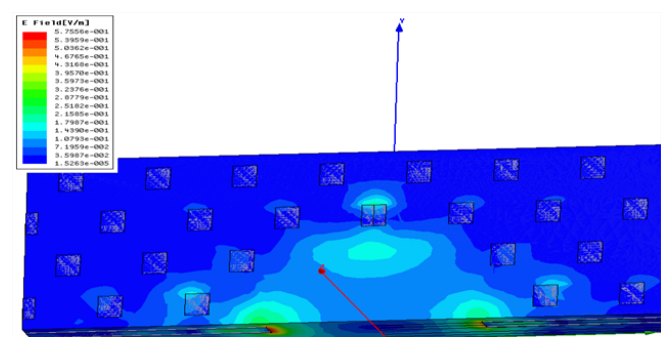

(b)

FIGURE 1. (a) A schematic of the woodpile structure designed for direct laser acceleration. (b) Magnitude of the longitudinal electric field for the accelerating mode. Symmetry of the structure was exploited using perfect magnetic boundary conditions on the bottom plane in order to reduce the simulation size to half of the complete structure.

The critical parameters for the design of a woodpile accelerator structure have been explored through simulations by Cowan [2]. These simulations provide the framework needed to guide the initial steps of fabrication. Structure parameters are dimensionless in simulations, scaling in proportion to the lattice constant, a, of the structure. The rod width, w, was determined in simulations by maximizing the bandgap. In order to maintain a face-centered cubic 


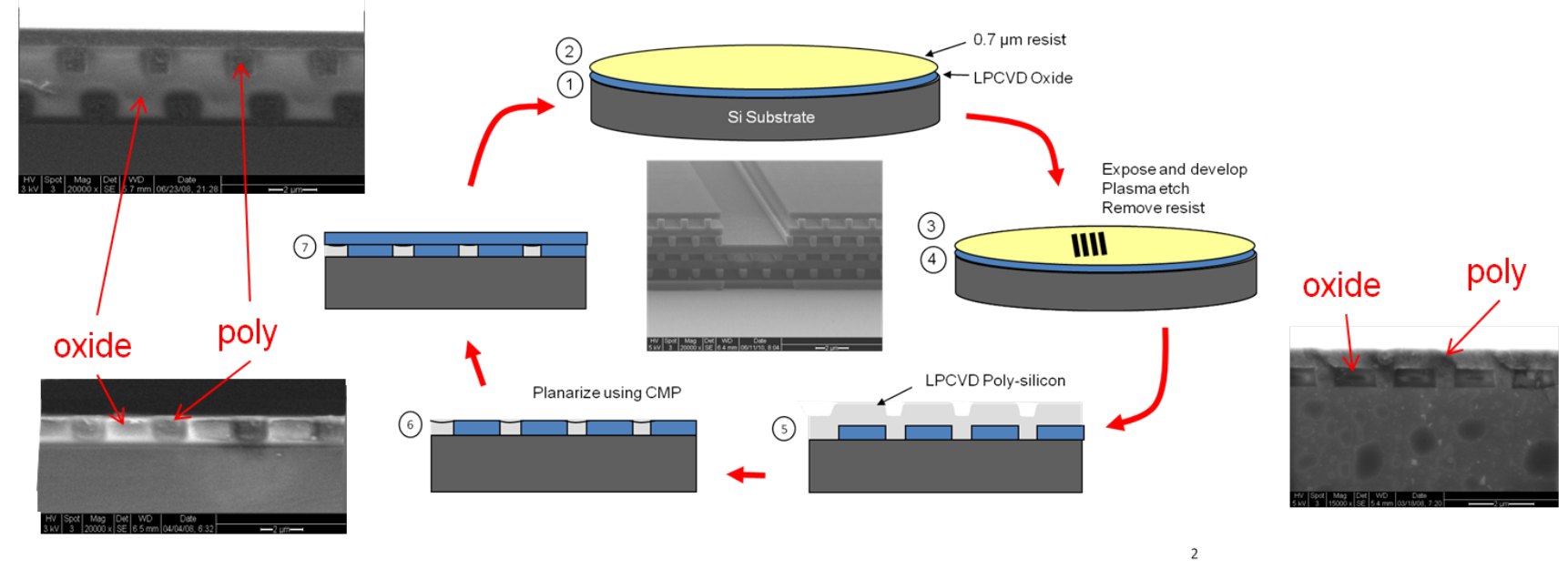

FIGURE 2. Schematic of the process flow developed for the fabrication of the woodpile accelerator structure. SEM images are included to supplement the schematic and provide verification of the process.

(FCC) crystal structure, the layer thickness, h, was held to $\frac{\sqrt{2}}{4} a$. The actual scale of the structure was limited by the minimum feature size achievable in the lithography process, setting a minimum rod width of $400 \mathrm{~nm}$. These dimensions correspond to a structure with a center bandgap wavelength of $4.26 \mu \mathrm{m}$. Ultimately the structure dimensions will be chosen to correspond to the wavelength that maximizes the damage threshold and accelerating gradient. In this paper we discuss the process used to fabricate an eight and nine layer structure, spectroscopy measurements of the resulting structure, and a comparison of these measurements to simulations.

\section{FABRICATION}

The fabrication process, depicted in Figure 2, is based on a process described by Lin and Flemming [6]. This method uses standard semiconductor processing techniques in a layer-by-layer approach, where each layer is deposited as a thin film, patterned using optical lithography, and etched to remove the undesired material. Each layer is planarized, using oxide as a support material for the silicon features, and successive layers are deposited onto the previous ones. The oxide is selectively removed once the desired number of layers are complete. Recently, more advanced methods have been developed in order to achieve smaller features, better alignment, more layers, or to reduce the number of steps required for a given number of layers. For example, using direct laser writing [7, 8] one can pattern an entire three dimensional structure, with a minimum feature size on the order of $100 \mathrm{~nm}$, in a single step. This reduces the fabrication time significantly. The laser writing process restricts the initial structure material to certain types of resist, and a complicated inversion process using atomic layer deposition (ALD) must be used to produce structures of other materials. Wafer fusion bonding is another method used for various substrate materials such as GaAs, InP, or Si, and feature sizes below $180 \mathrm{~nm}$ have been achieved using this method [9, 10]. This approach relies on a sophisticated interferometric alignment and fusion bonding technique. While the Lin-Flemming method used here is not as exotic as some of these methods, each of the steps in the process are reliable and the tools for each step are readily available at a number of nanofabrication facilities.

The specific details of the Lin-Flemming process have been modified and refined over several iterations. Double side polished wafers are used in order to balance the film deposition on both sides of the wafer, and prevent bowing of the wafer due to stress. A layer of silicon dioxide is deposited using low pressure chemical vapor deposition (LPCVD). Silane $\left(\mathrm{SiH}_{4}\right)$ and oxygen are flowed across the wafers in a furnace heated to $400{ }^{\circ} \mathrm{C}$ at $200 \mathrm{mTorr}$. A reaction of the form

$$
\mathrm{SiH}_{4}+\mathrm{O}_{2} \rightarrow \mathrm{SiO}_{2}+2 \mathrm{H}_{2}
$$

occurs at temperatures above $300{ }^{\circ} \mathrm{C}$. Using a tylan furnace to control the temperature and pressure, the uniformity across a wafer on average was $2.1 \%$, and the consistency of the deposition rate was within $3 \%$. An accurate deposition 
rate is important because the thickness of the oxide film defines the height of each layer of the structure. The oxide film is then annealed at $1000{ }^{\circ} \mathrm{C}$ for one hour in a nitrogen purged environment. This additional anneal step helps drive out residual gasses such as hydrogen that could diffuse out during a subsequent deposition and degrade the quality of the interface between film layers [11].

Optical lithography for the first layer is performed in several steps. Megaposit SPR955-CM i-line resist is spincoated on the oxide surface to a thickness of $0.7 \mu \mathrm{m}$. The resist is exposed using ASML PAS 5500 i-line stepper, with manufacturer specifications of $400 \mathrm{~nm}$ minimum feature size and $60 \mathrm{~nm}$ alignment accuracy $(3 \sigma)$. Based on these specifications the structure was designed for rod widths of $400 \mathrm{~nm}$, setting a lower limit on the operating wavelength. The mask used for imaging is a negative of the desired pattern for each layer. The pattern is ultimately inverted in the silicon deposition. Consistency of the tool performance is checked prior to each exposure by running a focus-exposure matrix across a test wafer. Optimal exposure and focus are determined by analyzing feature size as a function of exposure intensity and focus. This precursor step compensates for day-to-day variations in the tool due to slight temperature variations, prior user settings, and changes induced by the increasing number of oxide layers. Once the resist is exposed, the wafers are developed in Microposit MF-26A developer and baked at $110^{\circ} \mathrm{C}$ for 90 seconds.

The pattern exposed in the resist is then etched into the oxide layer below. Applied Material's Precision 5000 uses a magnetically enhanced reactive ion etch (MERIE) to create a highly anisotropic etch, resulting in optimal side wall profiles. The recipe was tuned by adjusting the flow rates of the gasses, pressure in the chamber, and magnetic field strength. Optimal side wall profiles were found for flow, pressure and magnetic field settings of $15 \mathrm{sccm} \mathrm{CHF}_{3}$, $30 \mathrm{sccm} \mathrm{CF} 4,100 \mathrm{sccm}$ Ar, 30 mTorr, and 60 Gauss. An additional 10\% over-etch is included in order to guarantee full contact between layers, and compensate for any etch nonuniformities. This results in an overlapping between layers, as can be seen in the final structure in Figure 3. Studies of overlapped woodpile structures show that a full bandgap still exists, and is effected minimally by a $10 \%$ overlap [12].

A layer of poly-silicon is then deposited upon the patterned and etched oxide layer. $\mathrm{SiH}_{4}$ gas undergoes pyrolysis (thermal decomposition) at high temperatures, resulting in a very uniform and conformal deposition. The LPCVD process is run at $620^{\circ} \mathrm{C}$ and a pressure of 400 mTorr. Rapid surface migration of poly-silicon in these conditions results in a uniform surface coverage, preventing any key-hole to form in the center of the etched channels. The polysilicon film completely fills the etched features, as well as the entire surface of remaining oxide, resulting in a profile depicted in the lower right of Figure 2.

Chemical mechanical polishing (CMP) both planarizes the wafer surface and removes the excess poly-silicon deposited. An end-point detector, which measures the friction in the polishing head is used to determine when the oxide surface is reached. A slight increase in friction is observed at this point allowing one to control the polishing to within $50 \mathrm{~nm}$. The initial LPCVD oxide deposition is targeted for an additional $45 \mathrm{~nm}$ in order to compensate for this slight over-polish, and the final layer thickness is typically within $25 \mathrm{~nm}$, or $5 \%$ of the target thickness. Both sides of the wafer are polished in order to balance the film stress on both sides of the wafer.

Once the layer is planarized the next layer is deposited, and the process repeats. After four layers of fabrication the wafers are annealed at $1100{ }^{\circ} \mathrm{C}$ in a nitrogen purged environment for $17 \mathrm{hrs}$. This helps reduce intrinsic stress in the

(a)

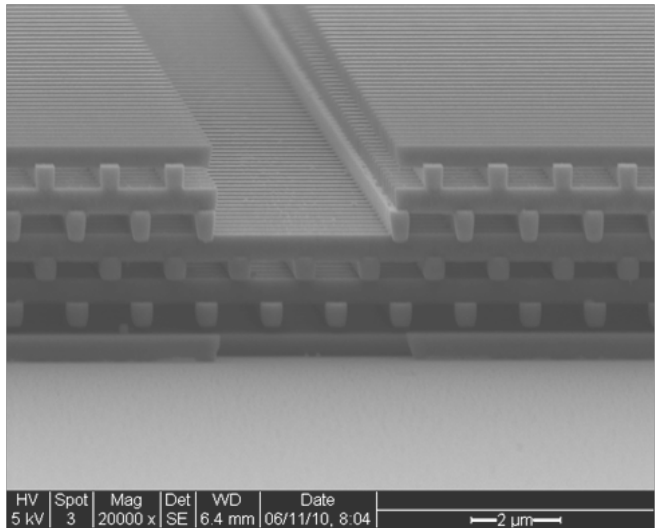

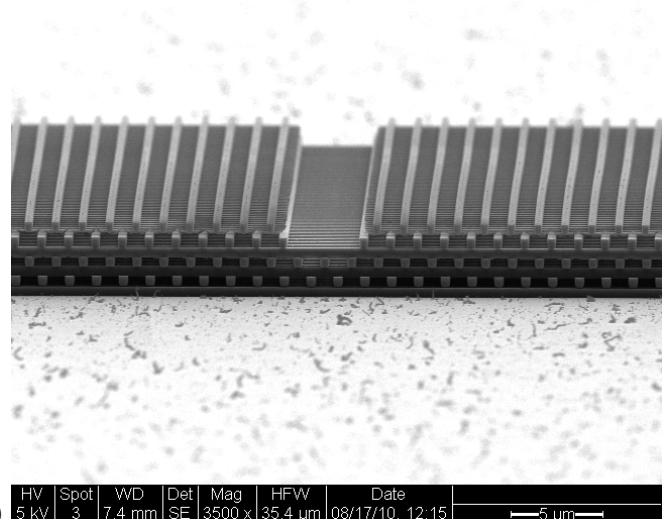

(b)

FIGURE 3. (a) A completed nine layer woodpile structure with a defect. The $5 \mu \mathrm{m}$ wide channel down the center of the structure is the bottom half of the defect. (b) A completed eight layer woodpile structure. This half of the structure will be aligned and bonded to the nine layer structure in order to achieve the complete seventeen layer structure. 
poly-silicon features which can cause delamination between layers. When the desired number of layers are complete, the oxide is removed using a hydrofluoric (HF) vapor etch. Aqueous HF etches induce strong capillary forces in the narrow trenches between each rod and often destroy the fragile structures, while the HF vapor etch does not.

\section{RESULTS}

Figure 3 shows completed 8 and 9 layer structures. Each of these structures represent one half of the complete seventeen layer structure, and will be aligned and bonded to complete the fabrication. Critical structure parameters such as layer thickness and rod width measured throughout the fabrication process were used both to monitor the process in order to make improvements, and to compile a set of parameters for use in simulations. The layer thickness is generally within $5 \%$ of the target while the rod width was initially $20 \%$ larger than target. The expansion in rod width occurs after the features are etched into the oxide, and is presumed to be due to contraction of the oxide at high temperatures during the poly-silicon deposition, causing the trenches to expand. This problem was compensated for upstream by adjusting the exposure such that the rod width patterned into the resist was less than the target rod width. SEM images were also taken of four layer structures after fabrication and the various structure parameters were measured a posteori. Figure 4 shows the resulting statistical data, again demonstrating the large overshoot in rod width in the first four layers.
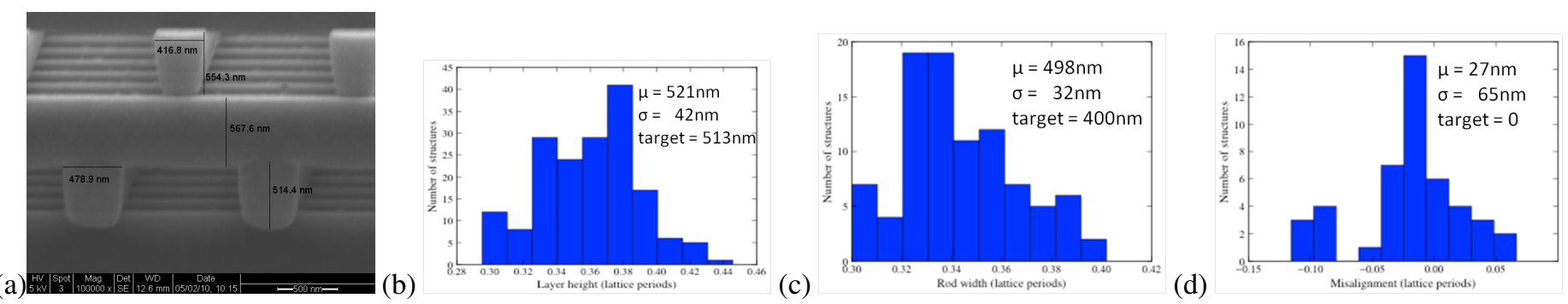

FIGURE 4. SEM images were taken for a large number of four layer structures, and statistical distributions were produced. The resulting histograms provide a set of fabrication tolerances achieved in this round of fabrication. The data shows high accuracy in layer thickness and alignment, but a large overshoot in rod width. These values provide a basis for simulations assessing the effect of fabrication tolerances on the photonic properties of the structure.

To characterize the quality of the fabricated photonic crystal and measure the effect of these variations, spectroscopy measurements were taken. These measurements were performed using a Nicolet 6700 Fourier transform infrared spectrometer (FTIR). A temperature controlled source produces a broad output from 3-25 $\mu \mathrm{m}$, which is focused onto the sample through a reflecting Cassegrain objective with a numerical aperture of 0.58 . The reflected light is recollected using the same objective and an interferogram is produced. Figure 5 (a) shows a schematic of the measurement apparatus. The Fourier transform of the resulting interferogram yields the reflectivity of the sample as a function of frequency or wavelength. Reflection spectra for four, six, eight, and nine layer structures are shown in Figure 5 (b). A distinct bandgap can be seen spanning from 3 to $5 \mu \mathrm{m}$, becoming more defined for the increasing number of layers.

(a)

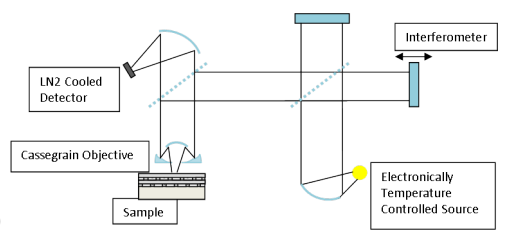

(b)

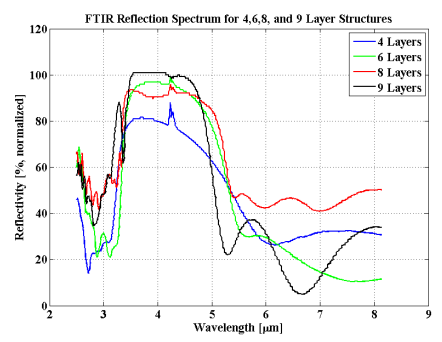

(c)

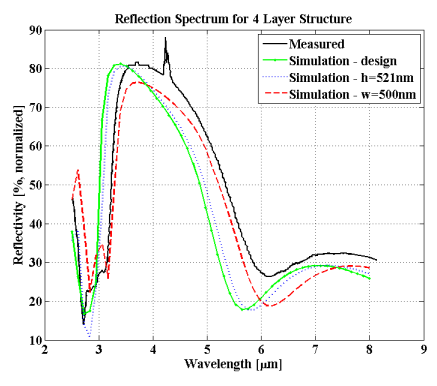

FIGURE 5. (a) Layout of the FTIR spectroscopy measurement aparatus. (b) Reflection spectrum for four, six, eight, and nine layer structures. (c) Comparison of reflection spectrum measured for a four layer structure, and results from simulations using both the designed structure parameters, and actual parameters measured in SEM images. Agreement in center bandgap wavelength is improved when accounting for the increased rod width in the fabricated structure. 
The measured reflection spectrum was analyzed by comparing it to simulations of the fabricated structure. Simulations were performed using a rigorous coupled-wave analysis (RCWA) code, in which eigenmode solutions for a given 2D plane are computed, assuming an infinite plane with a periodically modulated index of refraction. The field solutions for each plane are propagated from layer to layer using the scattering matrix method [13]. This code is uniquely suited to simulating this problem because it does not assume an infinite 3D lattice but rather a series of infinite 2D planes stacked on top of one another. In addition, this code allows one to model a structure with variations between successive layers. The code was benchmarked against MIT's Photonic Bands (MPB) by simulating an ideal structure with four, eight, and twelve layers and verifying the overlap of the bandgap region. To more closely approximate the measurement method, simulations for incident plane waves averaged over 10 polar angles and both $\mathrm{S}$ and $\mathrm{P}$ polarizations were computed. Figure 5 (c) shows the simulation results, compared to the measured reflection spectrum. The structure parameters in the simulation were modified according to the values measured, and the agreement with the measured value improves considerably when the increased rod width is included. Agreement in the center bandgap wavelength, determined by taking the center of the FWHM of the reflection peak, is within $3 \%$ when accounting for the measured structure parameters. Qualitative agreement in the features at the shorter and longer wavelength ends of the spectrum can also be seen, though are difficult to compare quantitatively. These results validate the quality of the photonic crystal and the fabrication process.

\section{CONCLUSION}

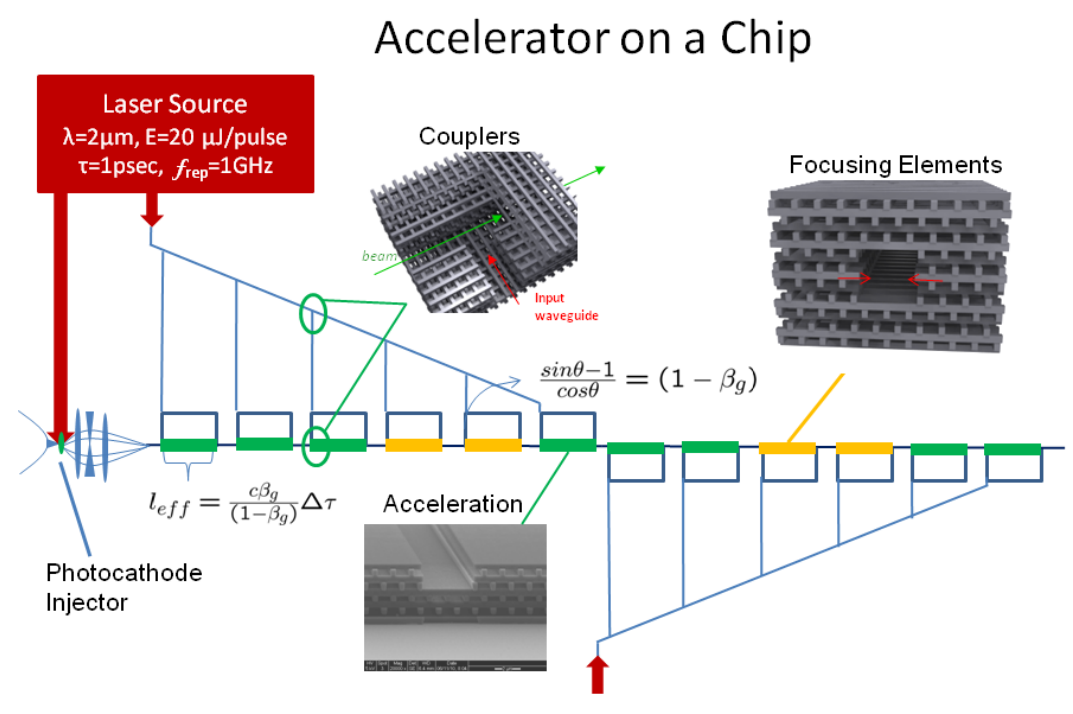

FIGURE 6. A schematic for a direct laser accelerator fabricated using lithographic techniques. Each accelerator segment is limited in length by the group velocity of the accelerating mode, and the pulse length of the laser. These segments are incorporated into cavities in order to increase the efficiency. The work presented here focuses specifically on the accelerator component. Couplers, focusing elements, bends, and the electron source are crucial components that are currently being designed.

The ultimate goal for this project is to design an entire accelerator that can be fabricated using lithographic techniques. We present initial results on one component of this type of accelerator. Addition components such as focusing elements, couplers, and bends are currently being studied, and will be critical to the implementation of the concept. Figure 6 demonstrates the general concept for a segment of an accelerator based on this technology. The length of individual accelerating segments are limited by the group velocity of the accelerating mode, and the pulse length of the laser pulse. For typical values of $v_{g}=0.3 c$ and $\tau=1 \mathrm{psec}$, this length is $100 \mu \mathrm{m}$. High alignment accuracy achievable by current lithography techniques will be needed to meet the severe alignment tolerances required for aligning the numerous accelerating segments in this design. The efficiency can be increased significantly if the 
accelerating segments are incorporated into cavities that are filled by an external laser pulse [5], as depicted by the ring cavities in Figure 6. It has been shown that focusing modes exist in the woodpile structure if one inserts the central rods into the structure slightly [14]. Couplers are also being designed in order to couple transverse polarized modes generated by traditional lasers, into the longitudinal mode required for acceleration. Many details remain to be explored, but the first set of experiments are soon to be underway and will provide experience and validation for the models and designs currently proposed. The successful fabrication of the first prototype structure will be a crucial step towards the implementation of a particle accelerator on a chip.

\section{ACKNOWLEDGMENTS}

The author would like to thank the SNF staff for their help with the entire fabrication process, with particular thanks to Cesar Baxter, James Conway, Nancy Latta, Mahnaz Mansourpour, Jim McVittie, Ed Myers, Ray Seymour, John Shott, Gary Sosa, Maurice Stevens, Mary Tang, Uli Thumser, and Mario Vilanova. Thanks also to Anthony Serpry for developing the RCWA code used for the simulations and to Professor Mark Brongersma for the use of the FTIR used for the spectroscopy measurements. Work supported by Department of Energy contracts DE-AC02-76SF00515 (SLAC) and DE-FG03-97ER41043-II (LEAP).

\section{REFERENCES}

1. Lin, X. E. "Photonic bandgap fiber accelerator". Phys. Rev. STAB 2001, 4, 051301.

2. Cowan, B.M. "Three-dimensional dielectric photonic crystal structures for laser-driven acceleration". Phys. Rev. STAB 2008, 11,011301

3. Sears, C.M.S.; et. al. "Phase stable net acceleration of electrons from a two-stage optical accelerator structure". Phys. Rev. STAB 2008, 11, 101301.

4. Siemann, R. H. "Energy efficiency of laser driven, structure based accelerators". Phys. Rev. STAB 2004, 7, 061303.

5. Na, Y.C.N.; Siemann, R.H.; Byer, R.L. "Energy efficiency of an intracavity coupled, laser-driven accelerator pumped by an external laser". Phys. Rev. STAB 2005, 8, 031301.

6. Lin, S. Y.; Flemming, J. G.; et. al. "A three-dimensional photonic crystal operating at infrared wavelengths". Nature 394 251-253, (1998).

7. Tetreault, N., et. al., "New route to three-dimensional photonic bandgap materials: silicon double inversion of polymer templates". Adv. Mater. 18 457-460 (2006).

8. Gratson, G., et. al., "Direct-write assembly of three-dimensional photonic crystals: conversion of polymer scaffolds to silicon hollow-woodpile structures". Adv. Mater. 18 461-465 (2006).

9. Noda, S., et. al., "Full three-dimensional photonic bandgap crystals at near-infrared wavelengths". Science 289 604-606, (2000).

10. Noda, S. "Recent progresses and future prospects of two- and three-dimensional photonic crystals". Journal of Lightwave Technology 2006, 24, 12, 4554-4567.

11. X. Ahang, K.S. Chen, S.M.spearing, "Thermo-mechanical behavior of thick PECVD oxide films for power MEMS applications". Sensors and Actuators A 103 263-270 (2003).

12. A. Feigel, B. Sfez, "Overlapped woodpile photonic crystals". Applied Optics 43, 4, (2004).

13. Tikhodeev, S.G.; Yablonskii, A.L.; Muljarov, E.A.; Gippius, N.A.; Ishihara, T. "Quasiguided modes and optical properties of photonic crystal slabs". Phys. Rev. B 2002, 66, 045102.

14. Cowan, B.M. "Photonic crystal laser-driven accelerator structures". Ph.D. Dissertation, Stanford University, Palo Alto, CA, 2007. 